Z. Phys. Chem. 216 (2002) 479-485

(C) by Oldenbourg Wissenschaftsverlag, München

\title{
Spatiotemporal Mixed-Mode Oscillations on a Ring Electrode
}

\author{
By Jaeyoung Lee, Johannes Christoph, Markus Eiswirth* and Gerhard Ertl \\ Fritz-Haber-Institut der Max-Planck-Gesellschaft, Faradayweg 4-6, D-14195 Berlin, \\ Germany
}

Dedicated to Prof. Dr. Friedemann W. Schneider

(Received October 11, 2001; accepted October 21, 2001)

\section{Pulse Reversal / Spatiotemporal Mixed-Mode Oscillations / Farey Sequence}

We present spatiotemporal mixed-mode oscillations in the electrocatalytic oxidation of formic acid on a platinum ring electrode modified by bismuth deposition. On the anodic scan, we observed spontaneous reversal of direction of a rotating activation pulse occurring repeatedly at the same location of the ring. In the constant potential method, spatiotemporal mixed-mode oscillations of pulse reversal were shown to constitute a fraction of an ordered Farey sequence.

\section{Introduction}

Nonlinear dynamical systems theory offers powerful tools for the analysis of experimentally observed complex phenomena exhibited by dissipative systems held far from equilibrium. In the 1980s, several studies have concentrated on temporal behaviour consisting of large and small oscillations with a substantial range of intermediate amplitudes unrealised [1-5]. This oscillatory phenomenon is referred to as mixed-mode oscillations (MMOs). Most sequences of different MMOs identified can be assigned to one of two classes: (a) periodic-chaotic sequences, in which intervals of periodicity are separated by an interval of chaotic states which resemble random mixtures of the adjacent periodic patterns, and (b) Farey sequences, i.e., periodic sequences for which a one-to-one correspondence with on ordered sequence of rational numbers can be established.

Hudson et al. [6,7] first observed periodic-chaotic sequences in the Belousov-Zhabontinskii (BZ) reaction. Swinney and Maselko [1,2] revealed

\footnotetext{
* Corresponding author. E-mail: eiswirth@ fhi-berlin.mpg.de
} 
a Farey sequence of MMOs. Farey sequences are familiar in periodically forced systems where periodic states are associated with locking of the driving frequency with an intrinsic frequency of the autonomous system. In a broader perspective, mixed-mode behaviour has been extensively studied within a nonlinear dynamics context. Albahadily et al. $[5,8]$ first reported similar behaviour for copper electro-dissolution in phosphoric acid, which is compared with similar occurrences reported for the Belousov-Zhabotinskii (BZ) reaction [2]. In the electrochemical system, the MMO scenario arises via a different nonlinear mechanism than the one proposed for the BZ reaction (locking on a torus). Ringland et al. [9] proposed a family of one-dimensional two-extremum maps as a limiting representation of the basic structure in phase-space that supports MMOs. The proposed mapping exhibits a spectrum of one-parameter sequences of MMOs, thus offering a strong basis for unification of the broad range of qualitatively different reported experimental observations of MMOs. Koper et al. $[10,11]$ proposed a simplified three-dimensional model of electrochemical MMOs processes taking place at rotating electrodes. The focus to date was on a set of states in which each member consists of a different combination of large and small temporal mixed-mode oscillations (TMMOs). This set of TMMOs was shown to constitute a Farey sequence, i.e., a periodic sequence for which a one-to-one correspondence exists with an ordered sequence of rational numbers. Spatiotemporal mixed-mode oscillations have been reported by Luss et al., who solved a model of heterogeneous catalysis [12,13].

One of the most prominent and stable patterns observed during formic acid oxidation on a Bi-modified Pt ring turned out to be a rotating pulse of electrocatalytic activity $[14,15]$. Here we describe experiments where such a rotating pulse occasionally reverses its direction spontaneously giving rise to spatiotemporal MMOs.

\section{Experimental}

A smooth polycrystalline Pt ring with inner diameter of $34.5 \mathrm{~mm}$ and outer diameter of $40.5 \mathrm{~mm}$ (thus exhibiting a geometric surface area of $7 \mathrm{~cm}^{2}$ ) was used as working electrode (WE). $\mathrm{A} \mathrm{Hg} / \mathrm{Hg}_{2} \mathrm{SO}_{4}$ reference electrode was placed at the center of the ring. A wire of platinized Pt served as counter electrode located well above $(40 \mathrm{~mm})$ the other electrodes. Twelve reference electrodes were distributed at about $300 \mu \mathrm{m}$ distance from the WE in order to monitor the local potential (see [14-18] for details).

A $1 \cdot 10^{-3} \mathrm{M} \mathrm{Bi}^{3+}$ containing solution was prepared by dissolution of highpurity bismuth (III) oxide $\left(\mathrm{Bi}_{2} \mathrm{O}_{3}\right.$, Strem Chemicals Inc., $\left.99.9998 \%\right)$ in $0.5 \mathrm{M}$ $\mathrm{HClO}_{4}$. Appropriate amounts of the $1 \cdot 10^{-3} \mathrm{M} \mathrm{Bi}^{3+}$ solution were added to the electrolyte to adjust the desired concentrations of $\mathrm{Bi}^{3+}$, which leads to a substantial increase of the electrocatalytic activity of $\mathrm{Pt}$ towards $\mathrm{HCOOH}$ oxidation. 
An in-house-built potentiostat (Electronic Lab of Fritz-Haber-Institut) was used for all $\mathrm{CV}$ and chronoamperometry (CA) experiments.

\section{Results and discussion}

In the electrocatalytic oxidation of $\mathrm{HCOOH}$ on a $\mathrm{Bi} / \mathrm{Pt}$ electrode, potentiostatic current oscillations in a potential range from $+110 \mathrm{mV}$ to $+280 \mathrm{mV}$ were observed [14-16]. In most of this range, a rotating pulse was observed [14, 15]. Towards higher overvoltage, it first became somewhat deformed (see Fig. 1), near the termination of current oscillations on the anodic scan during the cyclic voltammetry, spontaneous pulse reversal behavior was observed. Instead of increasing the potential in small steps, a similar sequence could be obtained in time because the gradual depletion of formic acid in front of the WE caused a slow parameter drift through the regime of interest. Thus, the potential was kept constant in order to measure traveling pulse reversal behavior as a function of time (chronoamperometry). Measured oscillations representing a variety of spatiotemporal mixed-mode states (SMMOs) are shown in Figs. 2-4.

The clear dichotomy between active pulse reversal and uni-directionally traveling pulse (uni-pulse) enables one to characterize a periodic state by listing the length of unbroken stretches of uni-pulse and reversed pulse in one period. In other words, for compactness, the periodic (non-chaotic) states are denoted as $\mathrm{P}^{\mathrm{R}}$, where $\mathrm{P}$ stands for the uni-directional pulse and $\mathrm{R}$ the pulse reversal on the ring of the particular spatiotemporal mixed-mode behaviour. This abbreviated notation is standard in the description of SMMOs. Thus, the list $\mathrm{P}_{1}, \mathrm{R}_{1}$, $\mathrm{P}_{2}, \mathrm{R}_{2}$, which could be written for the clarity in the form $\mathrm{P}_{1}{ }^{\mathrm{R}_{1}}-\mathrm{P}_{2}{ }^{\mathrm{R}_{2}}$, denotes
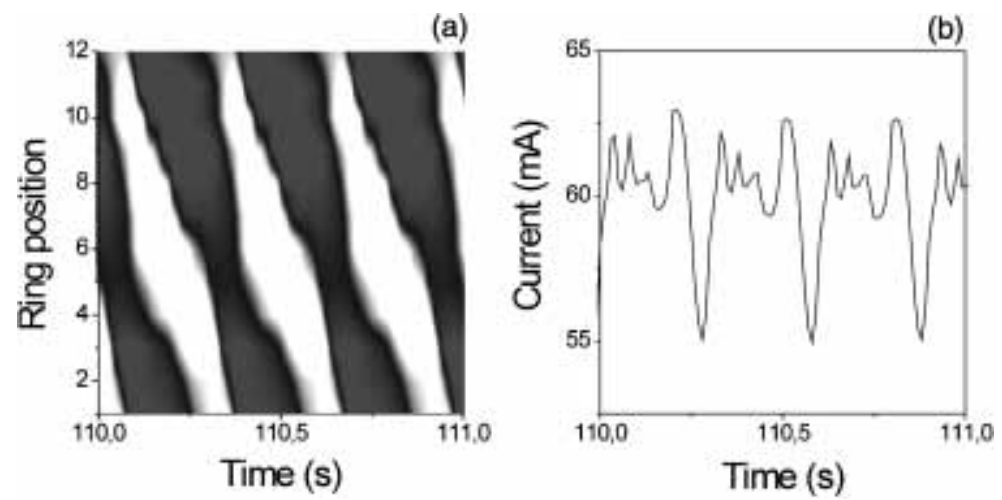

Fig. 1. (a) Spatial and (b) temporal oscillations of uni-pulse (always travelling with one direction along the ring; clockwise or counter-clockwise) during the potentiostatic oxidation of $\mathrm{HCOOH}$ on a $\mathrm{Bi} / \mathrm{Pt}$ ring electrode. $\mathrm{P}^{\mathrm{R}}=1^{0}, \beta=0.25$. Black and white colours indicate active and passive states of the electrode, respectively. 

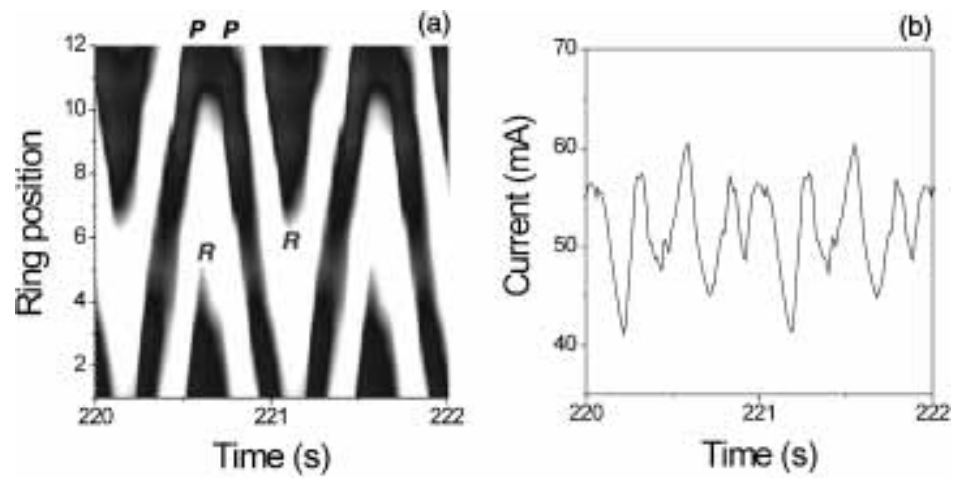

Fig. 2. (a) Spatial and (b) temporal mixed-mode oscillations of pulse reversal. The interfacial potential $(\phi)$ is measured with respect to a WE ring electrode $\left(\mathrm{P}^{\mathrm{R}}=1^{1}\right)$. Black and white colours indicate active and passive state of the electrode, respectively. $\boldsymbol{P}=$ uni-pulse and $\boldsymbol{R}=$ pulse reversal.
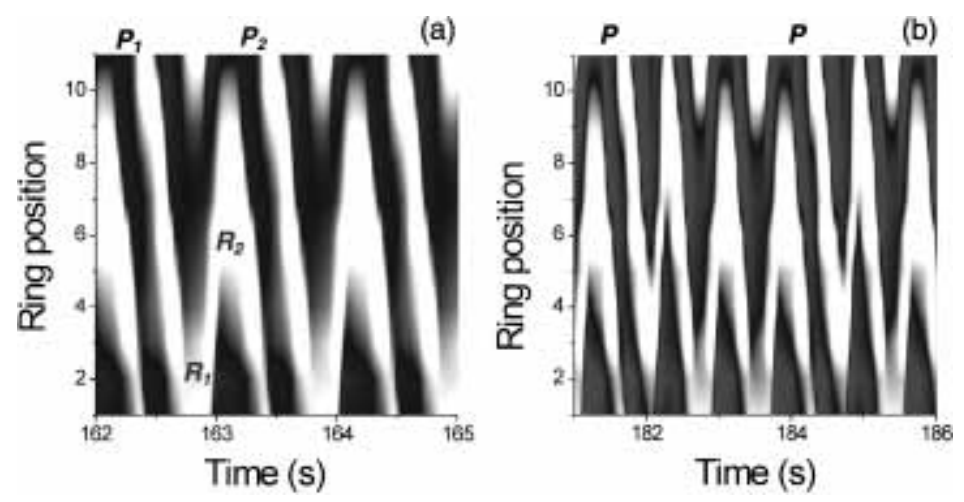

Fig. 3. Spatiotemporal patterns of (a) $\mathrm{P}^{\mathrm{R}}=1^{2}$ and (b) $\mathrm{P}^{\mathrm{R}}=1^{6}$ in the electro-oxidation of $\mathrm{HCOOH}$ on a $\mathrm{Bi} / \mathrm{Pt}$ surface $\left(\mathrm{P}^{\mathrm{R}}=1^{3}\right.$ not shown). Black and white colours indicate active and passive states of the electrode, respectively. $\boldsymbol{P}=$ uni-pulse and $\boldsymbol{R}=$ pulse reversal.

a state whose cycle consists of $\mathrm{P}_{1}$ uni-pulses followed by $\mathrm{R}_{1}$ pulse reversals, followed by $\mathrm{P}_{2}$ uni-pulses, followed by $\mathrm{R}_{2}$ pulse reversals.

The SMMOs shown in Figs. 1-4 indicate that the ratio of uni-pulse to pulse reversal steadily decreases with time.

Fig. 1 presents spatiotemporal mixed-mode oscillations of $1^{0}\left(\mathrm{P}^{\mathrm{R}}\right)$. A counter-clockwise active travelling pulse of interfacial potential is obtained along the ring. In Fig. 1b, asymmetric current oscillations, i.e., little spikes in one period, are observed, and it is clearly shown in the spatiotemporal behaviour. Between positions 3 and 7 the system is more passive (white domain) than at other positions. From the sequence observed in Fig. 1 and forthcoming 
(a)

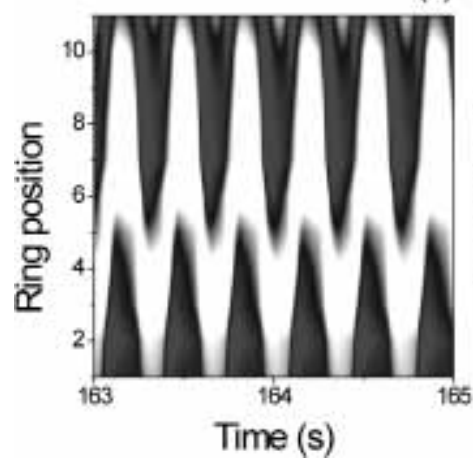

(b)

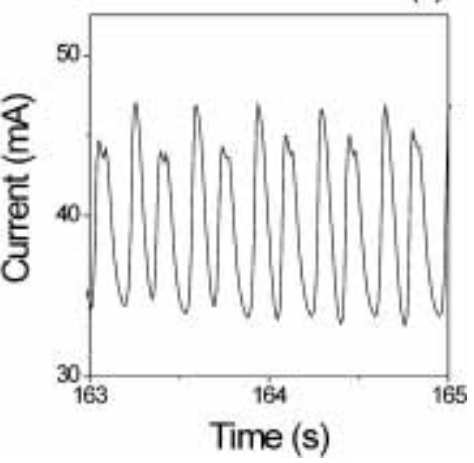

Fig. 4. (a) Spatial and (b) temporal mixed-mode oscillations of pulse reversal $\left(\mathrm{P}^{\mathrm{R}}=0^{1}\right)$ without any uni-direction pulse.

Figs. 2, 3, and 4, the sequence can be characterized as a fraction of a Farey tree. The Farey ratio in this case is defined as the ratio of uni-pulse to pulse reversal for a given type of behaviour.

Then, we first observe the behaviour consisting of one active uni-pulse followed by one pulse reversal, and it is a $1^{1}$ SMMOs (i.e., Farey ratio 1), as shown in Fig. 2a. This spatiotemporal pattern is clearly observed in the temporal current oscillations (see $\mathrm{P}$ and $\mathrm{R}$ in Fig. $2 \mathrm{~b}$ ). The state consisting of one uni-pulse followed by two pulse reversals (a $1^{2}$ SMMOs, Farey ratio 1/2), one uni-pulse followed by three pulse reversals (a $1^{3}$ SMMOs, Farey ratio $1 / 3$ ), and one uni-pulse followed by six pulse reversals (a $1^{6}$ SMMOs, Farey ratio 1/6) were subsequently also obtained (see Fig. 3).

During the experiments, we were also able to locate pure pulse reversals (a $0^{1} \mathrm{SMMO}$ ) in Fig. 4. In this case, the Farey ratio is 0.

The Farey tree arises in a number theory as an illustration of a scheme for the progressive generation of all the rational numbers between a given pair of rationals. This operation is known as Farey addition: the Farey sum of two rationals $a / c$ and $b / d$ is $(a+b) /(c+d)$; the ratios of the numerators and denominators are summed separately. The two starting rationals are written down at opposite sides of the page, and then each state of the process consists of writing their Farey sum between each pair of adjacent already-written numbers. A construction analogous to Fig. 5 follows if we spread the numbers out in the vertical dimension and draw a line segment from each number to both of its parents.

In Fig. 5 the spatiotemporal pulse reversal observed was listed. The list is laid out on an incomplete Farey tree, which manifests the relationships that exist among the states. It is seen that the pattern of each state is the concatenation of the patterns of a pair of states above it, one on either side. The state characterized by $1^{1}-1^{7}$ was the most complicated SMMO that could be reliably 


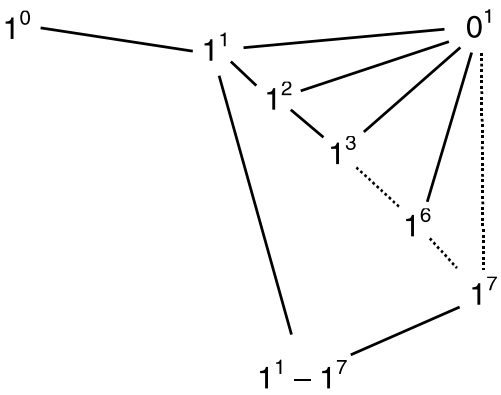

Fig. 5. A portion of the Farey tree constructed of observed states, the state $1^{7}$ was not obtained in experiment, though $1^{1}-1^{7}$ was.

reproduced in experiment. Before the transition to the $0^{1}$ state, in each experimental run also sequences $1^{1}-1^{n}$ with $n=10 \ldots 30$ were obtained, but those were never stable (usually only 1 period was obtained) and may actually have been due to parameter noise rather than being genuine attractors of the system. Examples are shown in Fig. 6.

The fact that spontaneous pulse reversal occurs is most likely due to an inhomogeneity of the ring electrode (located around positions 5 and 6). This

(a)

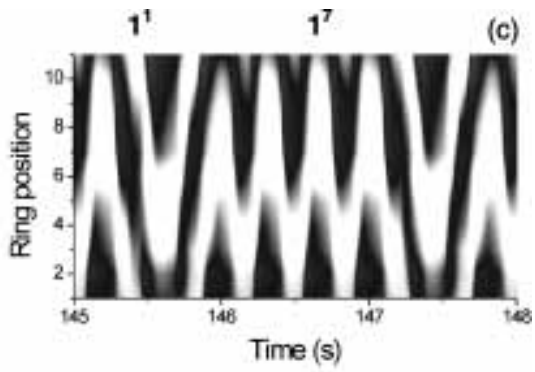

(b)

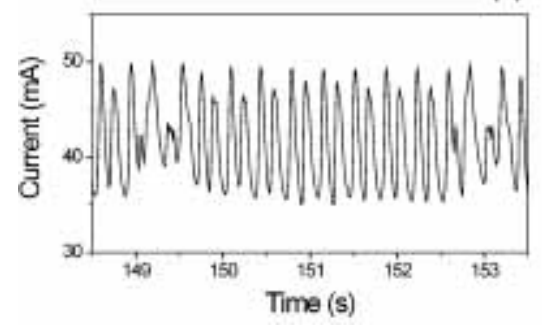

(d)

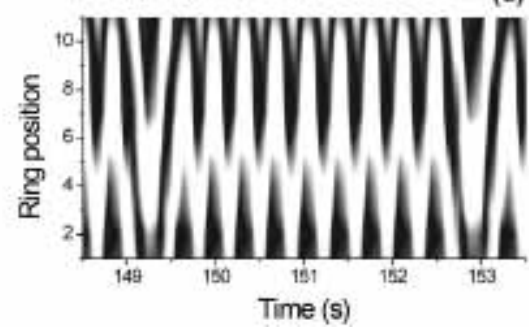

Fig. 6. Complex temporal mixed-mode oscillations (MMOs) of (a) $P_{1}{ }^{R_{1}}-P_{2}{ }^{R_{2}}=1^{1}-1^{7}$ and (b) $\mathrm{P}_{1}{ }^{\mathrm{R}_{1}}-\mathrm{P}_{2} \mathrm{R}_{2}=1^{1}-1^{17}$. Spatiotemporal MMOs of (c) $\mathrm{P}_{1}{ }^{\mathrm{R}_{1}}-\mathrm{P}_{2}{ }^{\mathrm{R}_{2}}=1^{1}-1^{7}$ and (d) $P_{1}{ }^{R_{1}}-P_{2}{ }^{R_{2}}=1^{1}-1^{17}$. Experimental conditions are the same as Fig. 1 . 
inhomogeneity (already visible in Fig. 1) can cause propagation failure of the pulse. However, due to the potentiostatic control, the active area is maintained constant (to a 1st approximation), i.e., the pulse cannot die so that it reverses direction upon propagation failure. In a system with local coupling only (e.g., a reaction-diffusion system) one would expect that the pulse either penetrates or reverses at the inhomogeneity $\left(1^{0}\right.$ or $0^{1}$ behavior). In contrast in an electrochemical (reaction-migration) system every point of the electrode is coupled (nonlocally) to every other one. Thus, the effect of an inhomogeneity depends on the state of the whole electrode (in particular on the pulse width) so that it becomes understandable that reversal may depend on the recent history of the pulse. In this way, the complicated mixtures of behavior in Figs. 2-4 can be attributed to the fact that the dynamics is a property of the whole electrode simultaneously rather than being determined locally.

\section{Acknowledgement}

Partial financial support by the Deutsche Forschungsgemeinschaft (DFG) through Sfb 555 is gratefully acknowledged.

\section{References}

1. H. L. Swinney and J. Maselko, Phys. Rev. Lett. 55 (1985) 2366.

2. J. Maselko and H. L. Swinney, J. Chem. Phys. 85 (1986) 6430.

3. R. Larter, C. L. Bush, T. R. Lonis and B. D. Aguda, J. Chem. Phys. 87 (1987) 5765.

4. P. DeKepper and K. Bar-Eli, J. Phys. Chem. 87 (1983) 480.

5. F. N. Albahadily, J. Ringland and M. Schell, J. Chem. Phys. 90 (1989) 813.

6. J. L. Hudson, M. Hart and D. Marinko, J. Chem. Phys. 71 (1979) 1601.

7. J. L. Hudson and J. C. Mankin, J. Chem. Phys. 74 (1979) 6171.

8. M. Schell and F. N. Albahadily, J. Chem. Phys. 90 (1989) 822.

9. J. Ringland, N. Issa and M. Schell, Phys. Rev. A 41 (1990) 4223.

10. M. T. M. Koper and P. Gaspard, J. Phys. Chem. 95 (1991) 4945.

11. M. T. M. Koper and P. Gaspard, J. Chem. Phys. 96 (1992) 7797.

12. U. Middya, M. Sheintuch, M. D. Graham and D. Luss, Physica D 63 (1993) 393.

13. U. Middya, D. Luss and M. Sheintuch, J. Chem. Phys. 100 (1994) 3568.

14. J. Christoph, P. Strasser, M. Eiswirth and G. Ertl, Science 284 (1999) 291.

15. P. Strasser, J. Christoph, W.-F. Lin, M. Eiswirth and J. L. Hudson, J. Phys. Chem. A 104 (2000) 1854.

16. J. Lee, J. Christoph, P. Strasser, M. Eiswirth and G. Ertl, J. Chem. Phys. 115 (2001) 1485.

17. J. Lee, Ph. D. Thesis, Freie Universität Berlin, Berlin 2001.

18. J. Lee, P. Strasser, M. Eiswirth and G. Ertl, Electrochim. Acta 47 (2001) 501. 